EPJ Web of Conferences 49, 13003 (2013)

DOI: $10.1051 /$ epjconf/20134913003

(C) Owned by the authors, published by EDP Sciences, 2013

\title{
Rare beauty and charm decays at LHCb
}

\author{
Johannes Albrecht ${ }^{1,2, a}$ on behalf of the LHCb collaboration \\ ${ }^{1}$ CERN, Geneva, Switzerland \\ ${ }^{2}$ TU Dortmund, Dortmund, Germany
}

\begin{abstract}
Rare heavy flavor decays are an ideal place to search for the effects of potential new particles that modify the decay rates or the Lorentz structure of the decay vertices. The LHCb experiment, a dedicated heavy flavour experiment at the LHC at CERN. It has recorded the worlds largest sample of heavy meson and lepton decays. The status of the rare decay analyses with $1 \mathrm{fb}^{-1}$ of $\sqrt{s}=7 \mathrm{TeV}$ and $1.1 \mathrm{fb}^{-1}$ of $\sqrt{s}=8 \mathrm{TeV}$ of $p p$-collisions collected by the LHCb experiment in 2011 and 2012 is reviewed. The worlds most precise measurements of the angular structure of $B^{0} \rightarrow K^{* 0} \mu^{+} \mu^{-}$and $B^{+} \rightarrow K^{+} \mu^{+} \mu^{-}$decays is discussed, as well as the isospin asymmetry measurement in $B \rightarrow K^{(*)} \mu^{+} \mu^{-}$decays. The first evidence for the very rare decay $B_{s}^{0} \rightarrow \mu^{+} \mu^{-}$ is presented together with the most stringent upper exclusion limits on the branching fraction of decays of $B^{0}$, $D^{0}$ and $K_{s}^{0}$ mesons into two muons. This note finishes with the discussion of searches for lepton number and lepton flavor violating $\tau$ decays.
\end{abstract}

\section{Introduction}

Flavor changing neutral current (FCNC) processes are forbidden at tree level in the Standard Model (SM), but can proceed via loop level electroweak penguin or box diagrams. In extensions to the SM, new virtual particles can enter in these loop level diagrams, modifying the decay rate or Lorentz structure of the decay vertex. Possible deviations from the SM predictions of these observables could lead to the discovery yet unknown phenomena. The search for these deviations is a complementary approach to direct searches at general purpose detectors and can give sensitivity to new particles at higher mass scales than those accessible directly.

This article reviews some of the most sensitive probes for possible extensions of the Standard Model that were measured by the $\mathrm{LHCb}$ collaboration at the time of the HCP conference (November 2012). Most measurements use a dataset of $1 \mathrm{fb}^{-1}$ of $\sqrt{s}=7 \mathrm{TeV}$ of $p p$-collisions collected in 2011. The search for $B_{s}^{0} \rightarrow \mu^{+} \mu^{-}$uses a combined dataset of $1 \mathrm{fb}^{-1}$ of $\sqrt{s}=7 \mathrm{TeV}$ and $1.1 \mathrm{fb}^{-1}$ of $\sqrt{s}=8 \mathrm{TeV}$, recorded in 2011 and 2012 .

The first part of the article discusses rare electroweak penguin transitions of the type ${ }^{1} b \rightarrow s \mu^{+} \mu^{-}$, which allow stringent tests of the Lorentz structure of the electroweak penguin processes. The second part discussed searches for purely leptonic decays of $K_{s}^{0}, D^{0}$ and $B$ mesons, which are particularly sensitive to new scalar interactions. The last class of analyses discussed is the search for lepton and baryon number violating $\tau$ decays.

\footnotetext{
a e-mail: albrecht@cern.ch

${ }^{1}$ In this proceedings, the inclusion of charge conjugate states are implicit, unless otherwise stated.
}

The implications of the presented measurements on possible extensions of the SM, most notably supersymmetric extensions, is discussed in a separate contribution in these proceedings [1].

\section{Electroweak penguin decays}

\subsection{Angular analysis and CP asymmetries in $B^{0} \rightarrow K^{* 0} \mu^{+} \mu^{-}$decays}

The decay $B^{0} \rightarrow K^{* 0} \mu^{+} \mu^{-}$has a branching fraction of $\mathcal{B}\left(B^{0} \rightarrow K^{* 0} \mu^{+} \mu^{-}\right)=\left(1.05_{-0.13}^{+0.16}\right) \times 10^{-6}$ [2]. It allows the construction of several observables with small hadronic uncertainties, that are sensitive to physics beyond the Standard Model (see [3, 4] and references therein). The LHCb collaboration performs an angular analysis in bins of the squared dimuon invariant mass $\left(q^{2}\right)$ and the three angles $\theta_{l}, \theta_{k}$ and $\phi$ [8]. $\theta_{l}$ is defined as the angle between the $\mu^{+}$ and the $B^{0}$ in the dimuon rest frame, $\theta_{k}$ as angle between the kaon and the $B^{0}$ in $K^{* 0}$ rest frame and $\phi$ as angle between the plane spanned by the dimuon system and the $K^{* 0}$ decay plane.

The differential branching ratio as a function of $q^{2}$ as well as the following observables have been measured (the observables are fully defined in [4, 9-11]): $A_{F B}$, the forward-backward asymmetry of the dimuon system; $F_{L}$, the fraction of $K^{* 0}$ longitudinal polarization; $S_{3}$, the transverse asymmetry, which is also often referred to as $\frac{1}{2}\left(1-F_{L}\right) A_{T}^{2}$ and $S_{9}$, a $C P$ averaged quantity corresponding to the imaginary component of the product of the longitudinal and transverse amplitudes of the $K^{* 0}$. The measurement of these observables is shown in Fig. 1, together with the SM prediction and the previous measurements of 

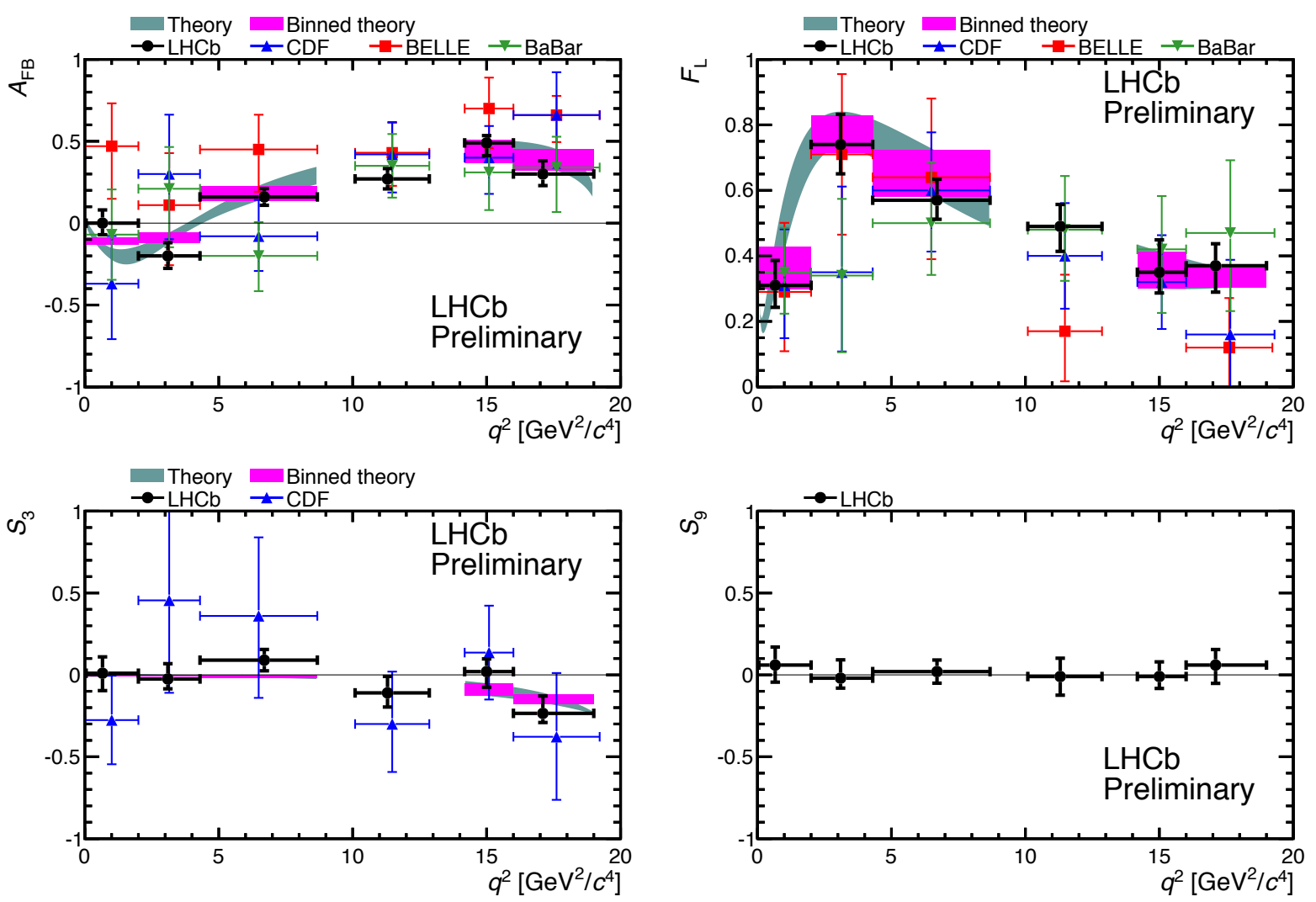

Figure 1. The observables $A_{F B}, F_{L}, S_{3}$ and $S_{9}$ measured in $B^{0} \rightarrow K^{* 0} \mu^{+} \mu^{-}$decays by the BABAR [5], Belle [6], CDF [7] and LHCb [8] experiments. The SM prediction, from Ref. [9], is also shown. Figure reproduced from Ref. [8].

other collaborations [5-8]. All observables are found to be consistent with each other and with the SM predictions. The $\mathrm{LHCb}$ results are the most precise measurements of these observables.

A particularly sensitive probe for new phenomena is $q_{0}^{2}$, the zero-crossing point of $A_{F B}$. It is theoretically very clean as the form factor uncertainties cancel at first order. The LHCb collaboration has reported the worlds first measurement as $q_{0}^{2}=4.9_{-1.3}^{+1.1} \mathrm{GeV}^{2} / \mathrm{c}^{4}$, in good agreement with the SM prediction. This measurement strongly disfavours scenarios with a flipped sign of the Wilson coefficient $C_{7}$.

The direct CP asymmetry in the $B^{0} \rightarrow K^{* 0} \mu^{+} \mu^{-}$system,

$$
\mathcal{A}_{C \mathcal{P}}=\frac{\Gamma\left(\bar{B}^{0} \rightarrow \bar{K}^{* 0} \mu^{+} \mu^{-}\right)-\Gamma\left(B^{0} \rightarrow K^{* 0} \mu^{+} \mu^{-}\right)}{\Gamma\left(\bar{B}^{0} \rightarrow \bar{K}^{* 0} \mu^{+} \mu^{-}\right)+\Gamma\left(B^{0} \rightarrow K^{* 0} \mu^{+} \mu^{-}\right)},
$$

is predicted to be of order $10^{-3}$ in the Standard Model. It was measured $1.0 \mathrm{fb}^{-1}$ of $7 \mathrm{TeV}$ data [12] to be

$$
\mathcal{A}_{C \mathcal{P}}=-0.072 \pm 0.040_{\text {stat }} \pm 0.005_{\text {syst }},
$$

integrated over the six $q^{2}$ bins. This measurement is consistent with the Standard Model prediction, it is the most precise measurement of $\mathcal{A}_{C \mathcal{P}}$ in $B^{0} \rightarrow K^{* 0} \mu^{+} \mu^{-}$decays to date.

\subsection{Angular analysis of $B^{+} \rightarrow K^{+} \mu^{+} \mu^{-}$decays}

The angular analysis of $B^{+} \rightarrow K^{+} \mu^{+} \mu^{-}$decays is performed analogously to the analysis of $B^{0} \rightarrow K^{* 0} \mu^{+} \mu^{-}$decays. The angular distribution of $B^{+} \rightarrow K^{+} \mu^{+} \mu^{-}$decays is given as [13]

$$
\frac{1}{\Gamma} \frac{d \Gamma}{d \cos \theta_{l}}=\frac{3}{4}\left(1-F_{H}\right)\left(1-\cos ^{2} \theta_{l}\right)+\frac{1}{2} F_{H}+A_{F B} \cos \theta_{l},
$$

where $A_{F B}$ denotes the forward backward asymmetry and $F_{H}$ the so called flat parameter. The SM predictions for both parameters are very small. Both $A_{F B}$ and $F_{H}$ can be significantly enhanced in models with large operators $C_{S}^{(\prime)}$ or $C_{P}^{(\prime)}$.

The LHCb collaboration has measured $A_{F B}$ and $F_{H}$ with $1 \mathrm{fb}^{-1}$ of data collected at $\sqrt{s}=7 \mathrm{TeV}$ [14], as shown in in Fig. 2. The measurement is found in good agreement with the SM predictions.

\subsection{Isospin asymmetry in $B \rightarrow K^{(*)} \mu^{+} \mu^{-}$}

The isospin asymmetry of the decays $B \rightarrow K^{(*)} \mu^{+} \mu^{-}, A_{I}$, is defined as

$$
A_{I}=\frac{\mathcal{B}\left(B^{0} \rightarrow K^{(*) 0} \mu^{+} \mu^{-}\right)-\frac{\tau_{0}}{\tau_{+}} \mathcal{B}\left(B^{+} \rightarrow K^{(*)+} \mu^{+} \mu^{-}\right)}{\mathcal{B}\left(B^{0} \rightarrow K^{(*) 0} \mu^{+} \mu^{-}\right)+\frac{\tau_{0}}{\tau_{+}} \mathcal{B}\left(B^{+} \rightarrow K^{(*)+} \mu^{+} \mu^{-}\right)},
$$



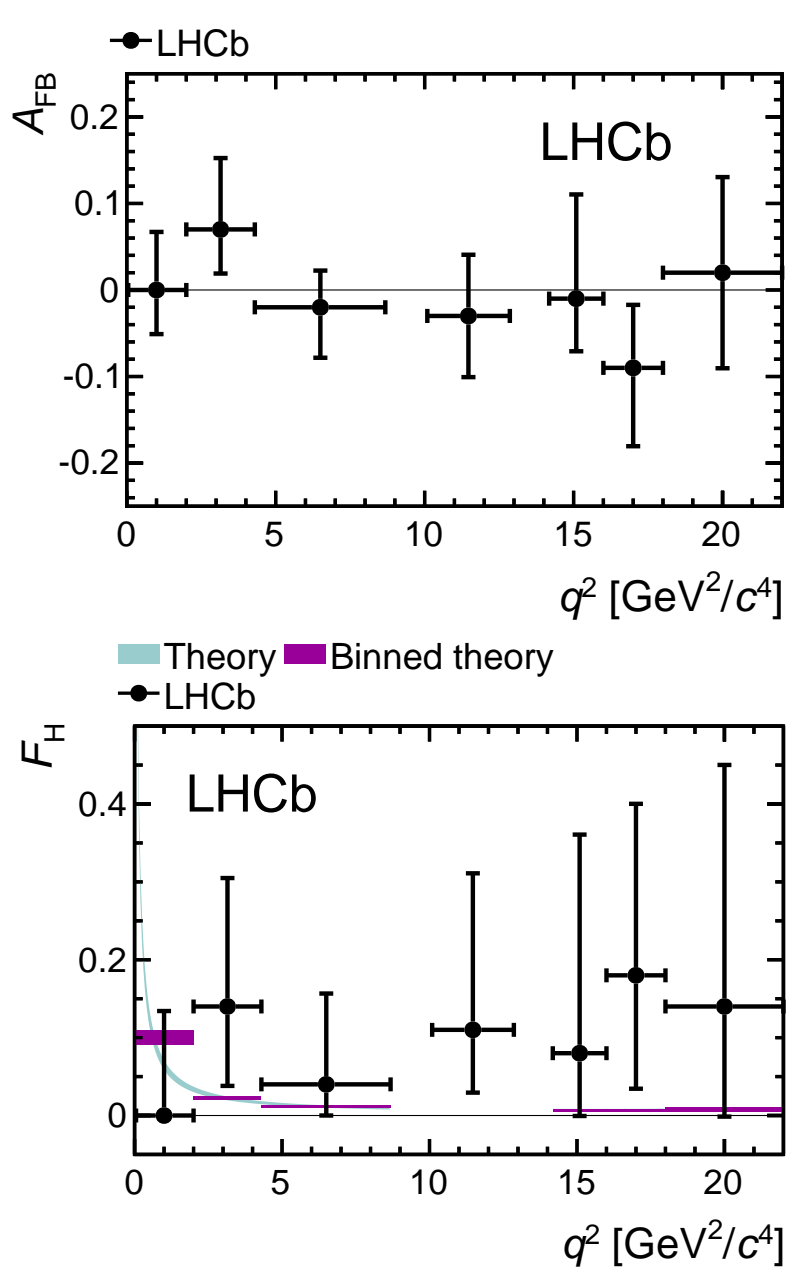

Figure 2. Dimuon forward-backward asymmetry, $A_{F B}$, and the parameter $F_{H}$ for $B^{+} \rightarrow K^{+} \mu^{+} \mu^{-}$as a function of the dimuon invariant mass squared, $q^{2}$.

where $\tau_{0,+}$ is the lifetime of the $B^{0}$ and $B^{+}$meson respectively. For the $B \rightarrow K^{*} \mu^{+} \mu^{-}$system, in the SM, $A_{I}$ is predicted to be -0.01 [15] with a slight increase at low values of $q^{2}$. For the $B \rightarrow K \mu^{+} \mu^{-}$system, the SM calculation of $A_{I}$ predicts a similar expectation close to zero [16]. The most precise measurement of $A_{I}$ is performed by the $\mathrm{LHCb}$ collaboration [17]. The measurement of $B \rightarrow K^{*} \mu^{+} \mu^{-}$is consistent with the SM prediction. The $B \rightarrow K \mu^{+} \mu^{-}$measurement shows a deviation from zero with a significance of greater than four standard deviations [17].

\section{Searches for very rare and forbidden decays}

\subsection{Searches in leptonic meson decays}

Decays of $K^{0}, D^{0}$ and $B_{s}^{0}$ or $B^{0}$ mesons into a muon pair are discussed in this section. The leptonic final state allows precise calculations of the expected rates and the two muon final state is experimentally a very clean signature. Both features together are making these decays very powerful tests of the Standard Model.

\subsubsection{Search for $K_{s}^{0} \rightarrow \mu^{+} \mu^{-}$}

The rare decay $K_{s}^{0} \rightarrow \mu^{+} \mu^{-}$can give insight into the shortdistance structure of $\Delta S=1 \mathrm{FCNC}$ transitions. This decay is highly suppressed in the SM, the predicted branching fraction is $\mathcal{B}\left(K_{s}^{0} \rightarrow \mu^{+} \mu^{-}\right)=(5.0 \pm 1.5) \times 10^{-12}[18,19]$. Contributions from possible extensions of the SM, e.g. from new light scalar particles, can enhance the branching fraction.

The LHCb dataset of $1 \mathrm{fb}^{-1}$ contains about $10^{13} K_{s}^{0}$ decays inside the detector acceptance. Signal candidates are separated from the background using BDT based selection. Main sources of residual background originate from semileptonic decays and $K_{s}^{0} \rightarrow \pi^{+} \pi^{-}$decays, where both pions are misidentified as muons. The latter can be separated from signal candidates exploiting the excellent mass resolution of the LHCb spectrometer. The contribution of $K_{L}^{0} \rightarrow \mu^{+} \mu^{-}$is found to be negligible for this analysis.

The number of expected signal events is evaluated using a relative normalization to $K_{s}^{0} \rightarrow \pi^{+} \pi^{-}$decays. This normalization reduces the systematic uncertainties which need to be considered in this analysis. The modified frequentist method $[20,21], \mathrm{CL}_{\mathrm{s}}$, is used to evaluate the consistency of the observed pattern of events with the background and signal plus background hypotheses. The expected upper exclusion limit is at 95\% $\mathrm{CL} \mathcal{B}\left(K_{s}^{0} \rightarrow\right.$ $\left.\mu^{+} \mu^{-}\right)<1.1 \times 10^{-8}$ and the observed limit is found to be $\mathcal{B}\left(K_{s}^{0} \rightarrow \mu^{+} \mu^{-}\right)<1.1 \times 10^{-8}$. This limit constitutes an improvement of a factor 30 with respect to the previous best limit.

\subsubsection{Search for $D^{0} \rightarrow \mu^{+} \mu^{-}$}

The $D^{0} \rightarrow \mu^{+} \mu^{-}$decay is predicted to be very rare in the Standard Model [22]: $1 \times 10^{-13}<\mathcal{B}\left(D^{0} \rightarrow \mu^{+} \mu^{-}\right)<$ $6 \times 10^{-11}$. This prediction can be significantly enhanced in MSSM scenarios with $\mathrm{R}$ parity violation, which predicts $\mathcal{B}\left(D^{0} \rightarrow \mu^{+} \mu^{-}\right) \sim 1 \times 10^{-9}$ mediated by a tree level transition [23].

The LHCb collaboration has performed an analysis using $0.9 \mathrm{fb}^{-1}$ of data at $\sqrt{s}=7 \mathrm{TeV}$ [24]. The background is reduced using a multivariate discriminant based on geometrical and kinematic information. The signal events are normalized to the $D^{* \pm} \rightarrow D^{0}\left(\rightarrow \pi^{+} \pi^{-}\right) \pi^{ \pm}$channel, which allows to reduce common systematic uncertainties. The event yield is determined from a two dimensional fit on the dimuon invariant mass and the difference between the $D^{* \pm}$ mass and $D^{0}$ mass. The observed pattern of events is compatible with the background expectations and an upper limit on the branching fraction of $\mathcal{B}\left(D^{0} \rightarrow \mu^{+} \mu^{-}\right)<1.3 \times 10^{-8}$ is determined at $95 \% \mathrm{CL}$, using the $C L_{s}$ method. This is the worlds most stringent limit on this decay.

\subsubsection{Evidence for $B_{s}^{0} \rightarrow \mu^{+} \mu^{-}$}

The search for the loop- and helicity suppressed decays $B_{s}^{0} \rightarrow \mu^{+} \mu^{-}$and $B^{0} \rightarrow \mu^{+} \mu^{-}$constitute a very stringent test of possible extensions of the SM, specially those with an extended scalar sector. The LHCb collaboration 
observes an excess of signal candidates in the channel $B_{s}^{0} \rightarrow \mu^{+} \mu^{-}$[25], which is inconsistent with the background hypothesis with a significance of 3.5 standard deviations. This measurement provides the first evidence for this decay, the measured branching fraction is consistent with the SM expectation. The $B_{s}^{0} \rightarrow \mu^{+} \mu^{-}$candidates with a high signal likelihood are shown in Fig. 3. The measurement is discussed in more detail in a separate contribution of these proceedings [26].

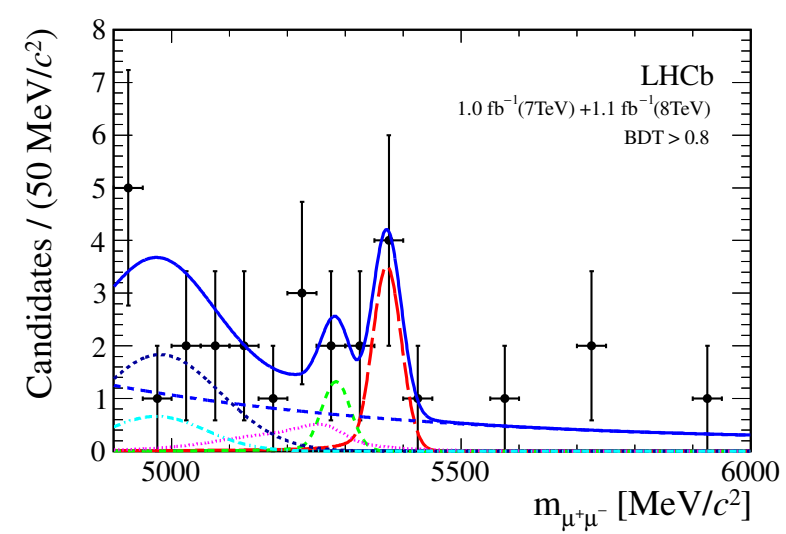

Figure 3. Invariant mass distribution of selected $B_{s}^{0} \rightarrow \mu^{+} \mu^{-}$ candidates (black points) with a high signal likelihood. The result of the fit is overlaid (blue line) and the different components detailed: $B_{s}^{0} \rightarrow \mu^{+} \mu^{-}$(red) and $B^{0} \rightarrow \mu^{+} \mu^{-}$(violet) SM signals, $B_{(s)}^{0} \rightarrow h^{+} h^{-}$(green), $B^{0} \rightarrow \pi^{-} \mu^{+} \nu_{\mu}$ (black) and $B^{0(+)} \rightarrow \pi^{0(+)} \mu^{+} \mu^{-}$(light blue) exclusive backgrounds.

\subsection{Search for forbidden $\tau^{-}$decays}

Lepton flavor violating (LFV) $\tau^{-}$decays only occur in the Standard Model from neutrino mixing. Many extensions beyond the SM predict enhancements, up to observable values which are close to the current experimental bounds.

\subsection{1 $\tau^{-} \rightarrow \mu^{+} \mu^{-} \mu^{-}$}

The neutrinoless decay $\tau^{-} \rightarrow \mu^{+} \mu^{-} \mu^{-}$is a particular sensitive mode in which to search for LFV at $\mathrm{LHCb}$ as the experimental signature with the three muon final state is very clean and the inclusive $\tau^{-}$production cross-section at $\mathrm{LHCb}$ is very large, about $80 \mu \mathrm{b}$. The composition of the $\tau^{-}$production can be calculated from the $b \bar{b}$ and $c \bar{c}$ [27] cross-sections measured at the LHCb experiment and the inclusive branching ratios $b \rightarrow \tau$ and $c \rightarrow \tau$ [28]. About $80 \%$ of the produced $\tau^{-}$-leptons originate from $D_{s}^{-}$decays.

LHCb has performed a search for the decay $\tau^{-} \rightarrow$ $\mu^{+} \mu^{-} \mu^{-}$using $1.0 \mathrm{fb}^{-1}$ of data [29]. The signal events are normalized to the $D_{s}^{-} \rightarrow \phi\left(\mu^{+} \mu^{-}\right) \pi^{-}$channel. The upper limit on the branching fraction was found to be

$$
\mathcal{B}\left(\tau^{-} \rightarrow \mu^{+} \mu^{-} \mu^{-}\right)<6.3 \times 10^{-8}
$$

at $90 \%$ C.L, determined using the $C L_{s}$ method. This has to be compared with the current best experimental upper limit from the Belle collaboration: $\mathcal{B}\left(\tau^{-} \rightarrow \mu^{+} \mu^{-} \mu^{-}\right)<$
$2.1 \times 10^{-8}$ at $90 \%$ C.L. The large integrated luminosity that will be collected by the upgraded LHCb experiment will provide a sensitivity corresponding to an upper limit of a few times $10^{-9}[30]$.

$$
\text { 3.2.2 } \tau^{-} \rightarrow p \mu^{-} \mu^{-} \text {and } \tau^{-} \rightarrow \bar{p} \mu^{+} \mu^{-}
$$

The large $\tau^{-}$sample can be exploited by searching for the baryon number and lepton number violating decays $\tau^{-} \rightarrow$ $p \mu^{-} \mu^{-}$and $\tau^{-} \rightarrow \bar{p} \mu^{+} \mu^{-}$. Both decays have $|B-L|=$ 0 which is predicted by many NP models. The analysis for these channels [31] follows closely that of the $\tau^{-} \rightarrow$ $\mu^{+} \mu^{-} \mu^{-}$mode as described above.

The observed pattern of events for the two decays $\tau^{-} \rightarrow p \mu^{-} \mu^{-}$and $\tau^{-} \rightarrow \bar{p} \mu^{+} \mu^{-}$is consistent with the background expectation and upper limits on the branching fraction of

$$
\begin{aligned}
& \mathcal{B}\left(\tau^{-} \rightarrow p \mu^{-} \mu^{-}\right)<4.6 \times 10^{-7} \text { and } \\
& \mathcal{B}\left(\tau^{-} \rightarrow \bar{p} \mu^{+} \mu^{-}\right)<3.4 \times 10^{-7}
\end{aligned}
$$

are obtained, using the $C L_{s}$ method. These are the first searches performed for these decays.

\section{Conclusion}

Most scenarios of physics beyond the Standard Model of particle physics predict measurable effects in the flavor sector, in particular in rare meson or lepton decays. No sign of physics beyond the Standard Model has yet been observed and stringent limits on its scale have been set.

An angular analysis of the rare electroweak penguin decays $B^{0} \rightarrow K^{* 0} \mu^{+} \mu^{-}$and $B^{+} \rightarrow K^{+} \mu^{+} \mu^{-}$has been performed as well as a measurement of the isospin asymmetry in $B \rightarrow K^{(*)} \mu^{+} \mu^{-}$decays. The measurements are of unprecedented precision and, besides the isospin asymmetry in agreement with the SM prediction.

Sensitive probes for NP are the purely leptonic decays of $B, D^{0}$ and $K_{s}^{0}$ mesons, all of which have been analysed by the LHCb collaboration. The first evidence on the decay $B_{s}^{0} \rightarrow \mu^{+} \mu^{-}$has been measured and the most stringent upper exclusion limits on the other decays have been obtained. The $\mathrm{LHCb}$ collaboration has also pioneered the analysis of lepton flavour violating $\tau^{-}$decays by performing the first of such searches at a hadron collider.

Most measurements presented in this proceedings use $1 \mathrm{fb}^{-1}$ of data collected at $\sqrt{s}=7 \mathrm{TeV}$, about one third of the total dataset recorded by the $\mathrm{LHCb}$ experiment. Updates of the analyses with significantly improved sensitivity are expected in the coming year and beyond.

\section{References}

[1] T. Blake, these proceedings (2013)

[2] J. Beringer et al. (Particle Data Group), Phys. Rev. D86, 010001 (2012)

[3] A. Ali, T. Mannel, T. Morozumi, Physics Letters B 273, 505 (1991) 
[4] W. Altmannshofer, P. Ball, A. Bharucha, A.J. Buras, D.M. Straub et al., JHEP 0901, 019 (2009), 0811. 1214

[5] S. Akar (BaBar collaboration), Talk at Lake Louise Winter institute (2012)

[6] Wei, J.-T. et al. (BELLE Collaboration), Phys.Rev.Lett. 103, 171801 (2009), 0904 . 0770

[7] T. Aaltonen et al. (CDF Collaboration), "Phys. Rev. Lett. 108 081807" (2011)

[8] LHCb collaboration, LHCb-CONF-2012-008 (2012)

[9] C. Bobeth, G. Hiller, D. van Dyk, JHEP 1007, 098 (2010), 1006.5013

[10] F. Kruger, J. Matias, Phys.Rev. D71, 094009 (2005), 21 pages, 16 figures. Minor typo in Eq. (4.8) corrected: version to appear in Phys. Rev. D Report-no: UAB-FT 560, hep-ph/0502060

[11] C. Bobeth, G. Hiller, G. Piranishvili, JHEP 0807, 106 (2008), 0805.2525

[12] R. Aaij et al. (LHCb Collaboration) (2012), 1210.4492

[13] A. Ali, P. Ball, L. Handoko, G. Hiller, Phys.Rev. D61, 074024 (2000), hep-ph/9910221

[14] R. Aaij et al. (LHCb Collaboration) (2012), 1209.4284

[15] T. Feldmann, J. Matias, JHEP 0301, 074 (2003), hep-ph/0212158
[16] A. Khodjamirian, T. Mannel, Y.M. Wang (2012), 1211.0234

[17] R. Aaij et al. (LHCb Collaboration), JHEP 1207, 133 (2012), 1205. 3422

[18] G. Ecker, A. Pich, Nucl.Phys. B366, 189 (1991)

[19] G. Isidori, R. Unterdorfer, JHEP 0401, 009 (2004), hep-ph/0311084

[20] T. Junk, Nucl.Instrum.Meth. A434, 435 (1999), hep-ex/9902006

[21] A.L. Read, Journal of Physics G: Nuclear and Particle Physics 28, 2693 (2002)

[22] G. Burdman et al., Phys.Rev. D66, 014009 (2002), hep-ph/0112235

[23] E. Golowich et al., Phys.Rev. D79, 114030 (2009), arXiv: 0903.2830

[24] LHCb collaboration, LHCb-CONF-2012-005 (2012)

[25] R. Aaij et al. (LHCb Collaboration), Phys. Rev. Lett. 110, 021801 (2013)

[26] J. Albrecht, these proceedings (2013), 1302 . 1317

[27] R. Aaij et al. (LHCb Collaboration), Eur.Phys.J. C71, 1645 (2011), 1103.0423

[28] K. Nakamura et al. (Particle Data Group), J. Phys. G 37, 075021 (2010)

[29] LHCb collaboration, LHCb-CONF-2012-015 (2012)

[30] I. Bediaga et al. (LHCb collaboration) (2012), 1208.3355

[31] LHCb collaboration, LHCb-CONF-2012-027 (2012) 